\title{
Management of orbital lymphangioma using intralesional injection of OK-432
}

\author{
Yasuhito Suzuki, Akira Obana, Yuko Gohto, Tokuhiko Miki, Hisasi Otuka, Yuichi Inoue
}

Department of

Ophthalmology, Osaka

City University

Medical School

Y Suzuki

A Obana

Y Gohto

T Miki

Department of

Ophthalmology,

Kashihara City

Hospital

H Otuka

Department of

Radiology, Osaka City

University Medical

School

Y Inoue

Correspondence to:

Dr Yasuhito Suzuki,

Department of

Ophthalmology, Osaka City

University Medical School,

Asahi-machi 1-4-3,

Abeno-ku, Osaka City,

545-8585, Japan

m5253565@

msic.med.osaka-cu.ac.jp

Accepted for publication

14 January 2000

\begin{abstract}
Aim-To treat orbital lymphangioma with an intralesional injection of $\mathrm{OK}-432$ (group A Streptococcus pyogenes of human origin).

Method-A 14 year old boy had a right orbital cystic lymphangioma. The visual acuity in the eye was $20 / 28$. In an initial treatment, $0.02 \mathrm{mg}$ of $\mathrm{OK}-432$, was injected into the tumour after aspiration of the fluid contents, but no effect was seen. The second treatment was performed with $0.04 \mathrm{mg}$ of OK-432.

Result-4 months later, the lesion had totally shrunk to fibrous tissue. The side effects were fever, a local inflammatory reaction lasting 3 days, and increased intraocular pressure, which was managed by draining the fluid contents. Visual acuity improved to $20 / 15$, and the visual field defect and restriction of eye movement seen before treatment disappeared. No recurrence was noted 1 year after treatment.

Conclusion-An intralesional injection of OK-432 shrunk the lymphangioma without functional disturbance and scar in the facial skin. OK-432 may be useful for orbital lymphangioma, but further studies are still warranted to determine efficacy, complications, and the optimal dose for safe treatment.

(Br f Ophthalmol 2000;84:614-617)
\end{abstract}

Lymphangioma is a congenital malformation of a lymph duct, which is commonly seen in the head, neck, or armpits by paediatricians. It is histologically benign but often infiltrates into surrounding tissues. Orbital lymphangioma is treated by surgical excision. Although many cases of successful surgical resection of orbital lymphangioma have been reported, ${ }^{1-4}$ the results are not always satisfactory. In particular, total resection of the orbital lymphangioma is difficult, and any injury to nerve or muscle tissue can decrease visual acuity or cause disturbance of eye movement. Moreover, postoperative cicatrisation is a cosmetic problem, and residual tumour cells pose a risk of recurrence. $^{1-3}$

In recent years, numerous studies have reported that an intralesional injection of OK-432 (Picibanil; Chugai Pharmaceutical Co, Tokyo), which was developed as an anticancer agent showing immunopotentiation, was effective against lymphangioma in the head, neck, and armpits. ${ }^{5-12}$ These reports encouraged us to use this method to treat an orbital lymphangioma in a teenage boy. As far as we can tell, this is the first report of a case that was treated by intralesional injection of OK-432.

\section{Methods}

PATIENT

A 14 year old boy complained of swelling in his right lower eyelid in April 1997, and was diagnosed orbital lymphangioma based on computed tomography and magnetic resonance imaging by a local doctor. Family and medical histories were unremarkable. The swelling temporarily disappeared following aspiration of the tumour contents, a blood-like serous fluid, but it recurred in December and aspiration was repeated twice. Two days after the third aspiration, the severest eyelid swelling and proptosis were observed, seemingly due to bleeding in the tumour, and the patient complained of double vision. He came to our department in January 1998.

His best corrected visual acuity was $20 / 28$ right eye and 20/20 left eye. The intraocular pressure was $24 \mathrm{~mm} \mathrm{Hg}$ right eye (with carteolol hydrochloride eye drops (2\% Mikelan, Otsuka Pharmaceutical Co, Tokyo) and isopropyl unoprostone eye drops (Rescula, Fujisawa Pharmaceutical Co, Osaka)), as well as $750 \mathrm{mg}$ a day of an orally administered carbonate dehydratase inhibitor, acetazolamide, and $18 \mathrm{~mm} \mathrm{Hg}$ left eye. Hertel's exophthalmometric measurements were $24 \mathrm{~mm}$ in the right eye and $14 \mathrm{~mm}$ in the left, indicating a marked proptosis (Fig 1). A Hess chart showed strong restriction of eye movement in all directions. Noted in the right fundus were tortuosity of the retinal arteries and veins and elevation of the fundus caused by the tumour pressing on the nasal periphery, which in turn induced a retinal fold in the macular region. There was no abnormality in the anterior segment of either eye and in the left fundus. Visual field testing revealed a depression of the inter-

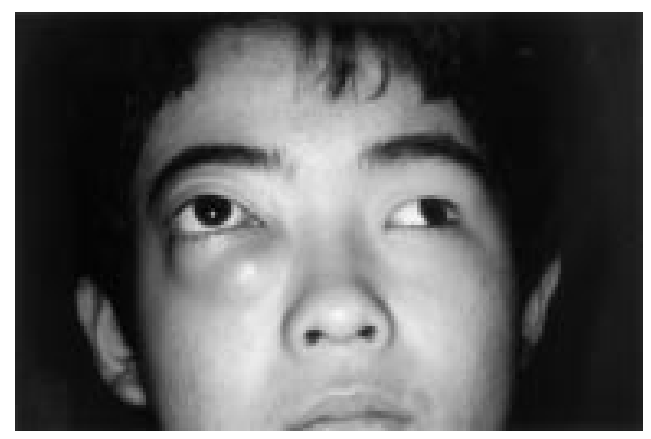

Figure 1 The patient before treatment. Note the distinct proptosis and the dislocation of the right eyeball laterally and upward. 
nal isoptre and a scotoma corresponding to the site of compression by the tumour in the right eye. A haematological examination was normal.

An orbital magnetic resonance image revealed a cystic tumour with a clear boundary extending from the nasal side of the eyeball to the retrobulbar muscle spindle and lower eyelid; the maximum transverse diameter was $40 \mathrm{~mm}$ and the length along the vertical axis 35 $\mathrm{mm}$ (Fig 2). The tumour showed high signals in both $\mathrm{T} 1$ and $\mathrm{T} 2$ weighted images, though they were slightly lower than those in the retrobulbar adipose tissue in the $\mathrm{T} 1$ weighted image. Inside the tumour, a structure resembling a septum was found. The eyeball, deformed by the tumour, protruded forward on the dorsal side. Ultrasound sonography revealed a cystic tumour with a septum.

\section{Treatment method and clinical course}

Before treatment, it was fully explained to the patient and his parents that the use of OK-432 in orbital lymphangioma had not been reported and the efficacy and complications were not known; signed informed consent was obtained according to the tenets of the Declaration of Helsinki. The first intratumour injection of OK-432 was given in February 1998. After disinfecting the skin with povidone iodine, a 24 gauge intravenous catheter was injected percutaneously into the tumour from the most swollen region inside the right lower
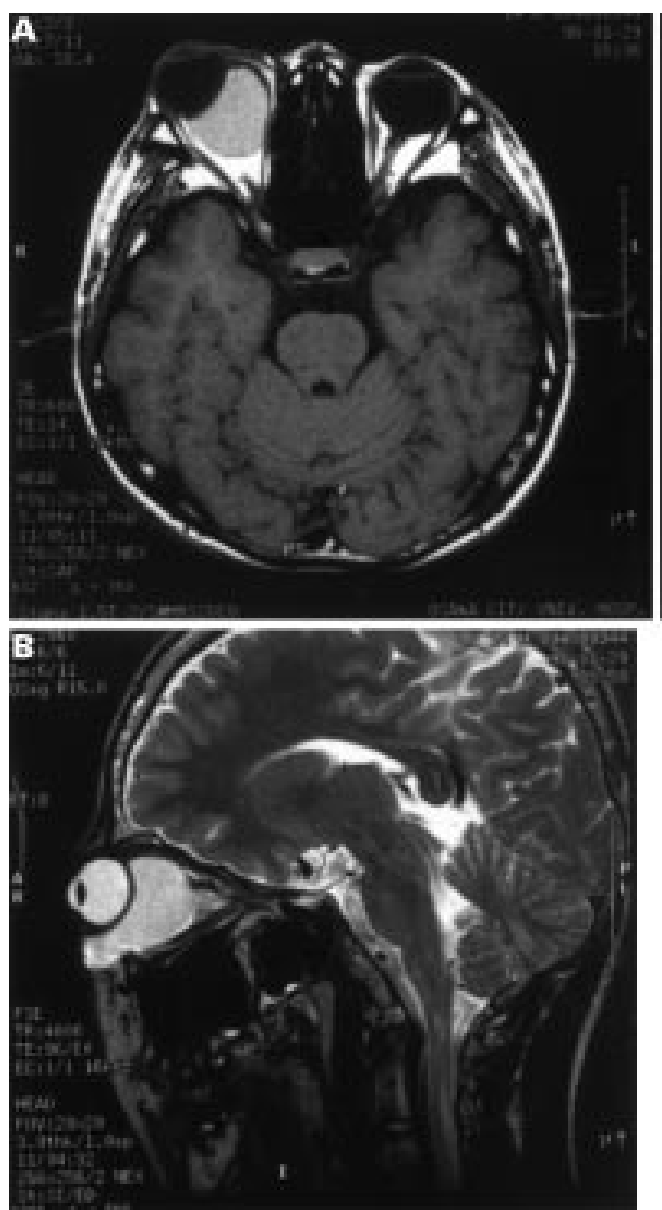

eyelid, and $11 \mathrm{ml}$ of the red-brown haematological fluid was aspirated, followed by an injection of $4 \mathrm{ml}$ of $0.005 \mathrm{mg} / \mathrm{ml}$ physiological saline solution $(0.02 \mathrm{mg})$ of OK-432. Cytodiagnosis of the aspirated fluid showed no malignant cells. Although the injection caused slight swelling and pain, there was no pyrexia or inflammatory reaction around the tumour. The appearance of the tumour 1 week after the injection was unchanged, and MRI showed no marked change in size and shape.

Because a sudden eyelid swelling, probably caused by bleeding inside the tumour, was noted on the 11 th day after the first injection, a second intratumour injection was administered that day; this time $13 \mathrm{ml}$ of the blood-like fluid was aspirated followed by an injection of $2 \mathrm{ml}$ of $0.02 \mathrm{mg} / \mathrm{ml}$ physiological saline solution $(0.04 \mathrm{mg})$ of OK-432. Five hours later, the patient complained of orbital pain and vomited. Although the pain was relieved temporarily by an intramuscular injection of an analgesic, orbital pain and marked swelling of the upper and lower eyelids as well as redness, local hot sensation, and conjunctival oedema recurred 15 hours later, and the intraocular pressure rose to $44 \mathrm{~mm} \mathrm{Hg}$ despite medical treatment. Then, $4 \mathrm{ml}$ of the contents were aspirated and IOP decreased to $12 \mathrm{~mm} \mathrm{Hg}$. However, because eyelid swelling and orbital pain appeared again after 3 hours, the fluid was aspirated again. Aspiration was performed three times within 3 days after the second

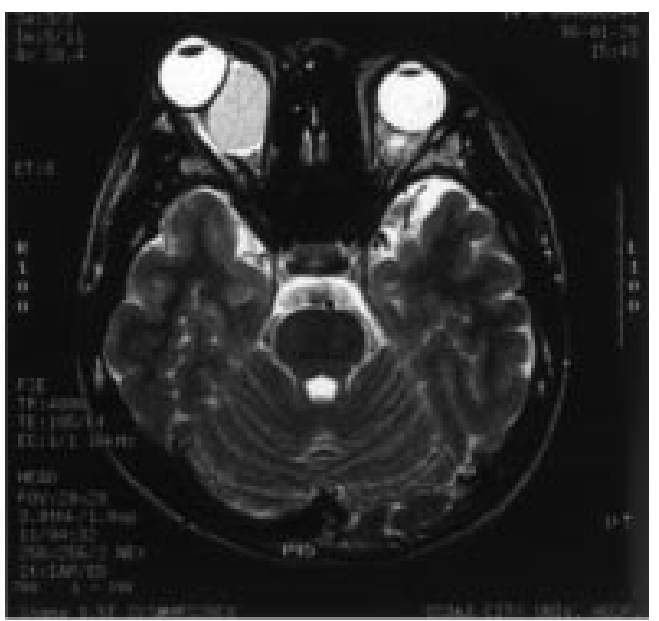

Figure 2 (A) T1 and T2 weighted images of a magnetic resonance image before treatment (horizontal section): note the high signals and septal structures inside. (B) T2 weighted images in sagittal section. A cystic tumour continues to the lower eyelid. 


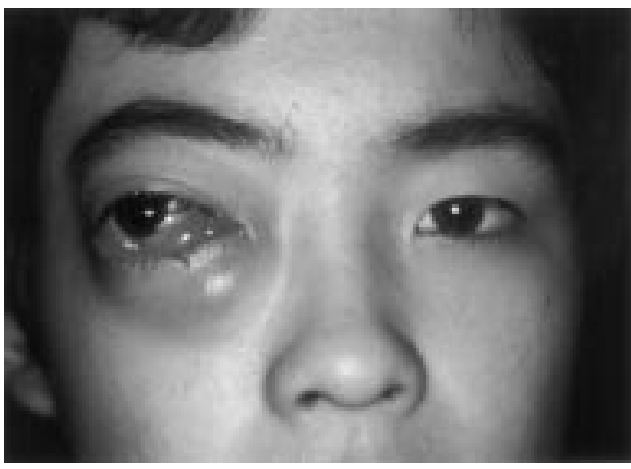

Figure 3 Swelling of the eyelid, redness, and conjunctival oedema are seen 7 days after the second intralesional injection of $\mathrm{OK}-432$.

injection, draining a total of $12.5 \mathrm{ml}$ of the contents. During this time, the maximum C reactive protein was 6.6 , the body temperature was $37.8^{\circ} \mathrm{C}$, and the white blood cell count was 11300 .

From the fourth to the 10th day, the IOP ranged between 10 and $20 \mathrm{~mm} \mathrm{Hg}$ with medication, and eyelid swelling and conjunctival oedema persisted (Fig 3). On the 23rd day after the injection, the intraocular pressure was $12 \mathrm{~mm} \mathrm{Hg}$ right eye without drugs, and eyelid swelling, redness, and conjunctival oedema were markedly diminished. Best corrected visual acuity was 20/15 right eye and 20/10 left eye. Proptosis had decreased to $19 \mathrm{~mm}$ in the right eye and $15 \mathrm{~mm}$ in the left. Eye movements became normal and double vision had disappeared. After 3 months, proptosis and eyelid swelling had vanished completely, and the tortuosity of the retinal blood vessels and retinal fold had improved. The visual field had normalised. A T2 weighted magnetic resonance image showed that the tumour was distinctly smaller, with a low signal, and that the fluid inside the tumour had disappeared, probably due to fibrosis (Fig 4). The extraocular muscle and optic nerve were normal. Twelve months after the second injection, the face was completely normal (Fig 5), and magnetic resonance imaging detected no recurrence of the tumour.
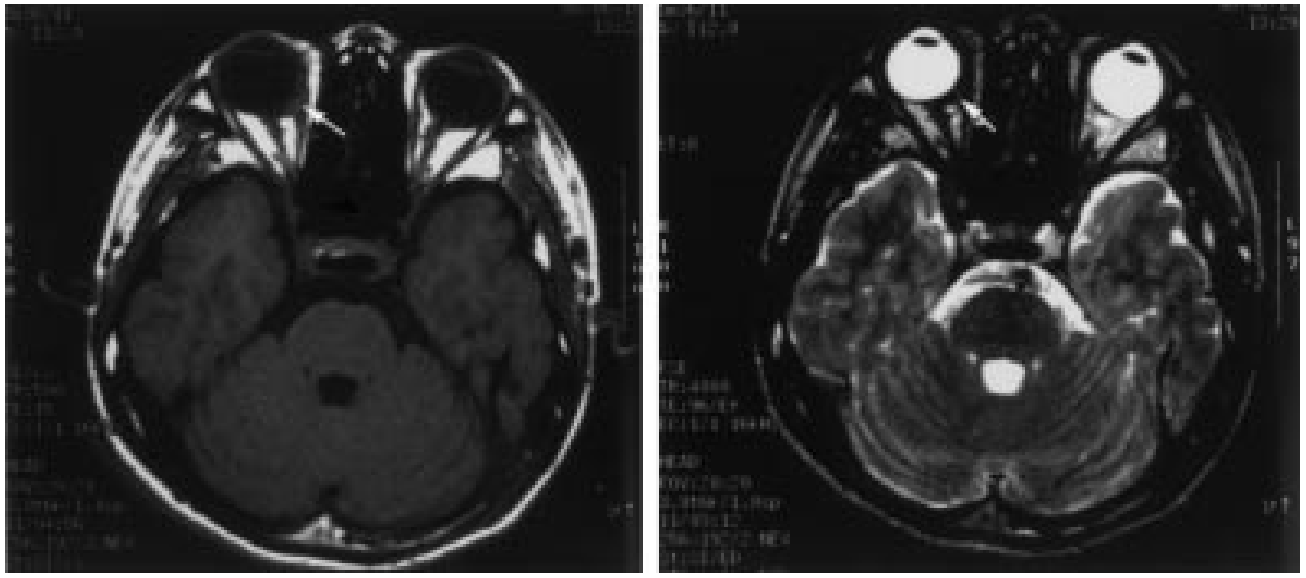

Figure $4 T 1$ and T2 weighted images from a magnetic resonance image done 4 months after the second intralesional injection of OK-432 demonstrate that the tumour had shrunk considerably and its aqueous component had disappeared.

\section{Discussion}

OK-432 is a preparation of dead bacteria developed by Okamoto et al. It is produced by incubating the culture of the low virulence Su strain of type III, group A Streptococcus pyogenes of human origin with penicillin $G$ potassium followed by lyophilisation of the incubation mixture, which results in the complete disappearance of streptolysin S producing ability. ${ }^{13}$ OK-432 has been used as an immunopotentiating anticancer drug since 1975. Also, having been shown to be effective in reducing and eliminating pleural effusion and ascites by local administration in patients with malignant accumulation, the drug is used by thoracic and abdominal surgeons. ${ }^{14} 15$

In 1986, Ogita et al for the first time performed intralesional injection of OK-432 to treat a lymphangioma in the right scapular region of a 4 month old girl. ${ }^{5}$ A local inflammatory reaction and temporary fever immediately followed the injection, but 50 days later the tumour had disappeared without a trace. Since then, many such clinical cases have been documented, with complete eradication or more than $50 \%$ regression of the lymphangioma in $67 \%$ of the cases. ${ }^{6}$ Specifically, the treatment was reported to be efficacious in $92 \%$ of cystic lymphangioma cases and in $44 \%$ of cavernous lymphangioma cases. ${ }^{6}$ The only adverse reactions have been temporary fever and local inflammatory reaction occurring in almost all the cases immediately after the drug administration for several days. The mechanism whereby intralesional injection of OK- 
432 causes regression of lymphangioma was considered to be destruction of the endothelial cells in the lymph duct by the inflammation induced by the drug. ${ }^{5}$ Subsequent detailed studies have suggested that injection of OK432 causes induction and activation of white blood cells which produce some kinds of cytokines. Those cytokines act on the endothelium of a lymphangioma to increase its permeability, and thus the accelerated lymph drainage and increased lymph flow led to shrinkage of the cystic space. ${ }^{16}$

In the present case no histological examination was performed, since biopsy of the lymphangioma was impossible because of haemorrhaging. However, lymphangioma was diagnosed clinically based on (1) characteristic MRI findings, ${ }^{17-19}$ (2) findings such as no orbital fracture, (3) an aspirated contents without malignant cells, and (4) the young age of the patient. The tumour was judged a cystic lymphangioma from ultrasound sonography and MRI findings.

Ogita et al recommended the use of physiological saline solution of OK-432 with a concentration from 0.005 to $0.02 \mathrm{mg} / \mathrm{ml}$, and a dosage of $0.2 \mathrm{mg}$ or less at one time. ${ }^{6}$ Satisfactory results have also been obtained using a concentration of $0.005 \mathrm{mg} / \mathrm{ml}$ solution. ${ }^{7}$ Based on that report, we administered $4 \mathrm{ml}$ of 0.005 $\mathrm{mg} / \mathrm{ml}$ solution $(0.02 \mathrm{mg})$ in the first session, but no effect was obtained. Ogita et al recommended readministration within 1 or 2 weeks if inflammatory findings such as fever and local swelling did not occur by the first or second day after the injection, and that administration be continued until these findings were observed. In our case, since no effects were observed until the 11 th day, a second injection was given. While injection of $2 \mathrm{ml}$ of 0.02 $\mathrm{mg} / \mathrm{ml}$ solution of OK-432 (0.04 mg) resulted in regression of the tumour, it was associated with distinct local swelling, pain, increased intraocular pressure, fever, and increased C reactive protein. Although it is reported that these inflammatory reactions are a sign of therapeutic success, in a lesion with a restricted space such as orbita, a distinct acceleration in exudation will increase the intraocular pressure, interfering with visual function. Therefore, further studies are required to determine if therapy with a lower dose, with in a milder reaction than in the present study, can still be effective.

Hypertonic glucose solution and bleomycin have been suggested as the drugs of choice for intralesional therapy against lymphangioma. However, there is a risk of infection with the former $^{20}$ and of pulmonary fibrosis with the latter, ${ }^{2122}$ and neither is widely used. In contrast, OK-432 injection does not cause serious systemic adverse reactions. The fact that the intralesional injection technique required is very simple and the outcome in the present case was satisfactory suggest that this is an alternative for the treatment of orbital lymphangioma. However, the present case is the first one to receive this treatment for orbital lymphangioma, and complications such as increased intraocular pressure as a result of local inflammation occurred. Therefore, we consider that this therapy is still at the experimental stage, and the further evaluation of its efficacy, complications, and dosimetry will be needed in many other cases before its widespread use.

1 Jackson WI, Green WR. Orbit lymphangiomas. Ophthalmology 1979;86:914-9.

2 Jones IS. Lymphangiomas of the ocular adnexa. Am $\mathcal{F ~ O p h -}$ thalmol 1961;51:481-509.

3 Harris GJ, Sakol PJ, Bonavolonta G. An analysis of thirty cases of orbital lymphangioma. Ophthalmology 1990;97: 1583-92.

4 Hemmer KM, Marsh JL, Milder B. Orbital lymphangioma. Plast Reconst Surg 1988;82:340-3.

5 Ogita S, Tsuto T, Takahasi T. A case report of sclerosing therapy with OK-432 for cystic hygroma in children. Geka therapy with OK-432 for
(Surgery) $1987 ; 49: 421-3$.

6 Ogita S, Tsuto T, Nakamura K, et al. OK-432 therapy for cystic hygroma and cavernous lymphangioma. $f_{p n} \mathcal{F}$ Pediatr Surg 1993;25:371-6.

7 Iwata M, Okabe I, Nonaka M, et al. The treatment of lymphangioma by local injection therapy with OK-432 in children. Fpn F Pediatr Surg 1993;25:377-83.

8 Ogita S, Tsuto T, Tokiwa K, et al. Intracystic injection of OK-432: a new sclerosing therapy for cystic hygroma in children. Br F Surg 1987;74:690-1.

9 Ogita S, Tsuto T, Deguchi E, et al. OK-432 therapy for unresectable lymphangiomas in children. 7 Pediatr Surg 1991;26:263-70

10 Watanabe K, Chiba T, Hatano G, et al. Intralesional OK-432 therapy for cystic hygroma. Otolaryngology: Head and Neck Surgery 1993;65:653-7.

11 Matsui R, Shiota H, Takeji $\mathrm{H}$, et al. A case of lymphangioma treated with intralesional OK-432. fpn f Clin Radiol 1997; 42:279-82.

12 Handa N, Asabe K, Kurosaka N. Intralesional injection of OK-432 for cystic lymphangioma. Ipn $\mathcal{F}$ Pediatr 1997;50: $1206-10$

13 Okamoto H, Shoin S, Koshimura S, et al. Studies on the and streptolysin S-forming abilities of hemolytic streptococci. fpn F Microbiol 1967;11:323-36.

14 Torisu M, Fujimura T, Kato M, et al. Successful immunotherapy of carcinomatous pleuritis and peritonitis by intracavitary injection of OK-432. In: Hoshino T, Uchda A, eds. Clinical and experimental studies in immunotherapy. Amsterdam: Excerpta Medica, 1983:99-112.

15 Nagao K. Studies on treatment of pleural carcinomatosis, with special reference to effect of OK-432. Chiba Med f 1982;58:345-53.

16 Ogita S, Tsuto T, Nakamura K, et al. OK-432 therapy for lymphangioma in children: Why and how does it work? $\mathcal{F}$ Pediatr Surg 1996;31:477-80.

17 Hasimoto M, Ohtsuka K, Nakagawa Y, et al. Magnetic resonance imaging of hemorrhagic orbital lymphangioma in a nance imaging of hemorrhagic orbital lympha
child. fpn $\mathcal{f}$ Clin Ophthalmol 1997;51:1481-4.

18 Bond JB, Haik BG, Taveras JL, et al. Magnetic resonance imaging of orbital lymphangioma with and without gadolinium contrast enhancement. Ophthalmology 1992;99: 1318-24.

19 Char DH, Sobel D, Kelly WM. Magnetic resonance scanning in orbital tumor diagnosis. Ophthalmology 1985; 92:1305-10

20 Chiba T, Ando T, Yaoita S, et al. Our experience on lymphangioma in children: with special reference to the sclerotherapy with high density glucose. $\mathcal{F} p n$ f Pediatr Surg 1989;21:445-9.

21 Ogino N, Okada A, Nakamura T, et al. Treatment for cystic ymphangioma with topical use of bleomycin. Fap f Pediat Surg 1984;16:925-30.

22 Nakajo T, Saeki M, Ogata T, et al. Cystic lymphangiomatreatments based on analysis of 273 cases. Fap $\mathcal{F}$ Pediat Surg 1984;16:931-8. 